\title{
On Semi-Pseudo-Ovoids
}

\author{
S. DE WINTER* \\ J.A. THAS \\ sgdwinte@cage.UGent.be \\ jat@cage.UGent.be \\ Department of Pure Mathematics and Computer Algebra, Ghent University, Krijgslaan 281-S22, B-9000 Gent, \\ Belgium
}

Received May 12, 2004; Revised December 7, 2004; Accepted December 7, 2004

Abstract. In this paper we introduce semi-pseudo-ovoids, as generalizations of the semi-ovals and semi-ovoids. Examples of these objects are particular classes of SPG-reguli and some classes of $m$-systems of polar spaces. As an application it is proved that the axioms of pseudo-ovoid $O(n, 2 n, q)$ in $\operatorname{PG}(4 n-1, q)$ can be considerably weakened and further a useful and elegant characterization of SPG-reguli with the polar property is given.

Keywords: semi-pseudo-ovoid, egg, SPG-regulus, polar property

\section{Introduction}

\subsection{Pseudo-ovals and pseudo-ovoids}

In $\mathrm{PG}(2 n+m-1, q)$ consider a set $O(n, m, q)$ of $q^{m}+1(n-1)$-dimensional subspaces $\mathrm{PG}^{(0)}(n-1, q), \mathrm{PG}^{(1)}(n-1, q), \ldots, \mathrm{PG}^{\left(q^{m}\right)}(n-1, q)$, every three of which generate a $\mathrm{PG}(3 n-1, q)$ and such that each element $\mathrm{PG}^{(i)}(n-1, q)$ of $O(n, m, q)$ is contained in a $\mathrm{PG}^{(i)}(n+m-1, q)$ having no points in common with any $\mathrm{PG}^{(j)}(n-1, q)$ for $j \neq i$. It is easy to check that $\mathrm{PG}^{(i)}(n+m-1, q)$ is uniquely determined, $i=0, \ldots, q^{m}$. The space $\mathrm{PG}^{(i)}(n+m-1, q)$ is called the tangent space of $O(n, m, q)$ at $\mathrm{PG}^{(i)}(n-1, q)$, $i=0, \ldots, q^{m}$. For $n=m$ such a set $O(n, n, q)$ is called a pseudo-oval or a generalized oval or an $[n-1]$-oval of $\mathrm{PG}(3 n-1, q)$; a generalized oval of $\operatorname{PG}(2, q)$ is just an oval of $\mathrm{PG}(2, q)$. For $n \neq m$ such a set $O(n, m, q)$ is called a pseudo-ovoid or a generalized ovoid or an $[n-1]$-ovoid or an egg of $\operatorname{PG}(2 n+m-1, q)$; a [0]-ovoid of $\operatorname{PG}(3, q)$ is just an ovoid of $\mathrm{PG}(3, q)$.

In Payne and Thas [9] (Theorem 8.7.2) it is proved that either $m a=n(a+1)$ or $n=m$, with $a$ an odd natural number, and that for $q$ even we have either $n=m$ or $2 n=m$. Also, in Payne and Thas [9] (Chapter 8) many other properties of $O(n, m, q)$ appear. It is still an open question whether or not for $q$ odd we have $m \in\{n, 2 n\}$.

Pseudo-ovals and pseudo-ovoids play an important role in the theory of finite generalized quadrangles, as in Payne and Thas [9] (Theorem 8.7.1) it is shown that their study is equivalent to the study of finite translation generalized quadrangles. 


\subsection{Semi-pseudo-ovoids}

A semi-pseudo-ovoid or a semi-egg of $\mathrm{PG}(h, q)$ is a non-empty set $O$ of $\eta$ mutually skew $(n-1)$-dimensional subspaces, denoted $\mathrm{PG}^{(i)}(n-1, q), i=1, \ldots, \eta$, with $h>2 n-1$, so that for every $i$ the union of all $n$-dimensional subspaces containing $\mathrm{PG}^{(i)}(n-1, q)$ and disjoint from $\mathrm{PG}^{(j)}(n-1, q)$, for every $j \neq i$, is an $(h-n)$-dimensional subspace $\mathrm{PG}^{(i)}(h-n, q)$ of $\mathrm{PG}(h, q)$. The space $\mathrm{PG}^{(i)}(h-n, q)$ is called the tangent space, or just the tangent, of $O$ at $\mathrm{PG}^{(i)}(n-1, q)$.

For $n=1$ semi-pseudo-ovoids are just semi-ovals and semi-ovoids; see Thas [11] and Buekenhout [2] for motivation, examples and existence.

It is also clear that pseudo-ovals and pseudo-ovoids provide examples of semi-pseudoovoids.

We now describe a method to construct a new semi-pseudo-ovoid from a given one. Let $O$ be a semi-pseudo-ovoid consisting of $(n-1)$-dimensional subspaces of PG $(h, q)$. Let $\pi \in O$ and assume that any $n$-dimensional subspace containing any element $\gamma$ of $O-\{\pi\}$ and any point of $\pi$, has a point in common with at least one element of $O-\{\pi, \gamma\}$. Then $O-\{\pi\}$ is still a semi-pseudo-ovoid of $\operatorname{PG}(h, q)$.

\section{The main inequalities}

\subsection{Main theorem}

Theorem 2.1 If $O$ is a semi-pseudo-ovoid consisting of $\eta(n-1)$-dimensional subspaces of $\mathrm{PG}(h, q)$, then

$$
1+q^{h-2 n+1} \leq \eta \leq 1+q^{\frac{h+1}{2}}
$$

It follows that $h \leq 4 n-1$.

Proof: Let $O=\left\{\pi_{1}, \pi_{2}, \ldots, \pi_{\eta}\right\}$ be a semi-pseudo-ovoid in $\operatorname{PG}(h, q)$ consisting of $\eta$ $(n-1)$-dimensional subspaces. The tangent space of $O$ at $\pi_{i}$ will be denoted by $\tau_{i}$, with $i=1,2, \ldots, \eta$. Further, let $\tilde{O}=\pi_{1} \cup \pi_{2} \cup \cdots \cup \pi_{\eta}$.

Consider an $n$-dimensional subspace $\beta$ with $\pi_{1} \subset \beta \subset \tau_{1}$ and let $\gamma$ be an $(n+1)$ dimensional subspace with $\beta \subset \gamma, \gamma \not \subset \tau_{1}$. Each $n$-dimensional subspace $\delta$ of $\gamma$ containing $\pi_{1}$, with $\delta \neq \beta$, contains a point of $\tilde{O}-\pi_{1}$. Hence

$$
\left|(\tilde{O} \cap \gamma)-\pi_{1}\right| \geq q
$$

There are exactly $\frac{q^{h-n}-1}{q-1}-\frac{q^{h-2 n}-1}{q-1}$ spaces $\gamma$. It follows that

$$
|\tilde{O}| \geq \frac{q^{n}-1}{q-1}+q \frac{q^{h-n}-q^{h-2 n}}{q-1},
$$


that is,

$$
|\tilde{O}| \geq \frac{q^{n}-1}{q-1}\left(1+q^{h-2 n+1}\right) .
$$

Consequently,

$$
|O| \geq 1+q^{h-2 n+1}
$$

Next, let $x_{i}$ be any point of $\operatorname{PG}(h, q)-\tilde{O}$, and let $t_{i}$ be the number of $n$-dimensional subspaces $\xi$ on $x_{i}$ with $\pi_{j} \subset \xi \subset \tau_{j}$ for some $j$. First we count the number of pairs $\left(x_{i}, \xi\right)$, with $x_{i} \in \xi, \xi n$-dimensional, and $\pi_{j} \subset \xi \subset \tau_{j}$ for some $j$. We obtain

$$
\sum_{i} t_{i}=\eta q^{n} \frac{q^{h-2 n+1}-1}{q-1}
$$

Next, we count the number of ordered triples $\left(x_{i}, \xi, \xi^{\prime}\right)$, with $x_{i} \in \xi, x_{i} \in \xi^{\prime}, \xi \neq \xi^{\prime}, \xi$ and $\xi^{\prime} n$-dimensional, $\pi_{j} \subset \xi \subset \tau_{j}$ for some $j$, and $\pi_{j^{\prime}} \subset \xi^{\prime} \subset \tau_{j^{\prime}}$ for some $j^{\prime}$. We obtain

$$
\sum_{i} t_{i}\left(t_{i}-1\right)=\eta(\eta-1) \frac{q^{h-2 n+1}-1}{q-1}
$$

Hence

$$
\sum_{i} t_{i}^{2}=\eta\left(\eta+q^{n}-1\right) \frac{q^{h-2 n+1}-1}{q-1}
$$

The number of points $x_{i}$ is equal to

$$
d=|\mathrm{PG}(h, q)-\tilde{O}|=\frac{q^{h+1}-1}{q-1}-\eta \frac{q^{n}-1}{q-1} .
$$

Now we have $d \sum_{i} t_{i}^{2}-\left(\sum_{i} t_{i}\right)^{2} \geq 0$, and so, by (2), (4) and (5)

$$
\left(\frac{q^{h+1}-1}{q-1}-\eta \frac{q^{n}-1}{q-1}\right) \eta\left(\eta+q^{n}-1\right) \frac{q^{h-2 n+1}-1}{q-1}-\left(\eta q^{n} \frac{q^{h-2 n+1}-1}{q-1}\right)^{2} \geq 0
$$

that is, $\eta^{2}-2 \eta-\left(q^{h+1}-1\right) \leq 0$, and so,

$$
\eta \leq 1+q^{\frac{h+1}{2}}
$$


Finally, from (1) and (6) follows that $1+q^{h-2 n+1} \leq 1+q^{\frac{h+1}{2}}$, and so

$$
h \leq 4 n-1 \text {. }
$$

\subsection{Pseudo-ovoids}

In this section we will show that by Theorem 2.1 the original definition of pseudo-ovoid $O(n, 2 n, q)$ can be considerably weakened.

Theorem 2.2 Iffor a semi-pseudo-ovoid $O$ consisting of $\eta(n-1)$-dimensional subspaces of $\mathrm{PG}(h, q)$ we have $h=4 n-1$, then $\eta=1+q^{2 n}$ and so $O$ is a pseudo-ovoid.

Proof: Assume that $h=4 n-1$ for the semi-pseudo-ovoid $O$. Then $h-2 n+1=$ $(h+1) / 2$, and so by Theorem 2.1 we have $\eta=1+q^{h-2 n+1}=1+q^{2 n}$. As we have equality in (6), we also have $d \sum_{i} t_{i}^{2}-\left(\sum_{i} t_{i}\right)^{2}=0$, with the notation of the proof of Theorem 2.1 . So $t_{i}$ is a constant. Hence

$$
t_{i}=\frac{\sum_{i} t_{i}}{d}=q^{n}+1
$$

for all $i$. As $\eta=1+q^{h-2 n+1}$, each $n$-dimensional subspace containing $\pi_{i} \in O$, but not contained in the tangent space of $O$ at $\pi_{i}$, contains exactly one point of $\tilde{O}-\pi_{i}$, where $\tilde{O}$ is the set of all points in all elements of $O$, with $i=1,2, \ldots, q^{2 n}+1$. It follows that any three distinct elements of $O$ generate a $(3 n-1)$-dimensional subspace of $\operatorname{PG}(h, q)$. Consequently $O$ is a pseudo-ovoid of $\operatorname{PG}(4 n-1, q)$.

\section{Remark}

(a) It follows that an egg $O(n, 2 n, q)$ is a set of $(n-1)$-dimensional subspaces of PG(4n$1, q)$, such that for each $\pi_{i} \in O(n, 2 n, q)$ the union of all $n$-dimensional subspaces containing $\pi_{i}$ but skew to all elements of $O(n, 2 n, q)-\left\{\pi_{i}\right\}$ is a $(3 n-1)$-dimensional subspace of $\mathrm{PG}(4 n-1, q)$.

(b) A weak egg of $\mathrm{PG}(4 n-1, q)$ is a set of $1+q^{2 n}(n-1)$-dimensional subspaces of $\mathrm{PG}(4 n-1, q)$, every three of which generate a $(3 n-1)$-dimensional subspace. It is an open question whether or not each weak egg is an egg; see Lavrauw [5].

\section{Interpretation of the equalities}

We will use the notation introduced in Section 2.

Theorem 3.1 For a semi-pseudo-ovoid $O$ we have $\eta=1+q^{\frac{h+1}{2}}$ if and only if each point not in an element of $O$ is on a constant number of tangent spaces. This constant equals $1+q^{\frac{h-2 n+1}{2}}$. 
Proof: Each point not in an element of $O$ is on a constant number of tangent spaces if and only if $t_{i}$ is a constant in the proof of Theorem 2.1, if and only if $d \sum_{i} t_{i}^{2}-\left(\sum_{i} t_{i}\right)^{2}=0$, if and only if $\eta=1+q^{\frac{h+1}{2}}$. In such a case

$$
t_{i}=\frac{\sum_{i} t_{i}}{d}=1+q^{\frac{h-2 n+1}{2}} .
$$

Theorem 3.2 For a semi-pseudo-ovoid $O$ we have $\eta=1+q^{\frac{h+1}{2}}$ if and only if each hyperplane not containing a tangent space of $O$, contains a constant number of elements of $O$. This constant equals $1+q^{\frac{h-2 n+1}{2}}$.

Proof: Let $\gamma_{i}$ be any hyperplane not containing a tangent space of the semi-pseudo-ovoid $O$. The number of elements of $O$ in $\gamma_{i}$ will be denoted by $u_{i}$. Now we count the number of pairs $\left(\gamma_{i}, \pi\right)$, with $\pi \in O$ in $\gamma_{i}$. We obtain

$$
\sum_{i} u_{i}=\eta q^{n} \frac{q^{h-2 n+1}-1}{q-1}
$$

Next we count the number of ordered triples $\left(\gamma_{i}, \pi, \pi^{\prime}\right)$, with $\pi \neq \pi^{\prime}, \pi \in O, \pi^{\prime} \in O$ and $\pi, \pi^{\prime}$ in $\gamma_{i}$. We obtain

$$
\sum_{i} u_{i}\left(u_{i}-1\right)=\eta(\eta-1) \frac{q^{h-2 n+1}-1}{q-1} .
$$

From (7) and (8) it follows that

$$
\sum_{i} u_{i}^{2}=\eta\left(\eta+q^{n}-1\right) \frac{q^{h-2 n+1}-1}{q-1} .
$$

The number of hyperplanes of $\mathrm{PG}(h, q)$ not containing a tangent space $\tau_{j}$ equals $\frac{q^{h+1}-1}{q-1}-$ $\eta \frac{q^{n}-1}{q-1}=g$. As $g \sum_{i} u_{i}^{2}-\left(\sum_{i} u_{i}\right)^{2} \geq 0$, we obtain

$$
\left(\frac{q^{h+1}-1}{q-1}-\eta \frac{q^{n}-1}{q-1}\right) \eta\left(\eta+q^{n}-1\right) \frac{q^{h-2 n+1}-1}{q-1}-\left(\eta q^{n} \frac{q^{h-2 n+1}-1}{q-1}\right)^{2} \geq 0,
$$

that is, $\eta^{2}-2 \eta-\left(q^{h+1}-1\right) \leq 0$, or equivalently,

$$
\eta \leq 1+q^{\frac{h+1}{2}} .
$$


We have equality in (10) if and only if $u_{i}$ is a constant. In such a case this constant equals

$$
u_{i}=\frac{\sum_{i} u_{i}}{g}=1+q^{\frac{h-2 n+1}{2}} \text {. }
$$

The theorem is proved.

Corollary 3.3 Iffor a semi-pseudo-ovoid $O$ we have $\eta=1+q^{\frac{h+1}{2}}$, then $\tilde{O}$ (Õ is the union of the elements of $O$ ) has two intersection numbers with respect to hyperplanes. Hence $\tilde{O}$ defines a projective linear two-weight code and a strongly regular graph.

Proof: If the hyperplane $\gamma$ contains a tangent space of $O$, then $\gamma \cap \tilde{O}$ is the disjoint union of one element of $O$ and $q^{\frac{h+1}{2}}(n-2)$-dimensional subspaces; if $\gamma$ does not contain a tangent space of $O$, then $\gamma \cap \tilde{O}$ is the disjoint union of $1+q \frac{h-2 n+1}{2}$ elements of $O$ and $q^{\frac{h+1}{2}}-q^{\frac{h-2 n+1}{2}}(n-2)$-dimensional subspaces. The fact that $\tilde{O}$ defines a projective linear two-weight code and a strongly regular graph now follows from Calderbank and Kantor [3].

Theorem 3.4 A semi-pseudo-ovoid $O$ is either a pseudo-oval or a pseudo-ovoid if and only if $\eta=1+q^{h-2 n+1}$.

Proof: Let $O=\left\{\pi_{1}, \pi_{2}, \ldots, \pi_{\eta}\right\}$ be a semi-pseudo-ovoid. Then, by the proof of Theorem 2.1, $\eta=1+q^{h-2 n+1}$ if and only if any $n$-dimensional subspace containing $\pi_{i}$, but not contained in the tangent space of $O$ at $\pi_{i}$, has exactly one point in common with $\tilde{O}-\pi_{i}$, for all $i=1,2, \ldots, \eta$, that is, if and only if any three distinct elements of $O$ generate a $(3 n-1)$-dimensional subspace, that is, if and only if $O$ is either a pseudo-oval or a pseudo-ovoid.

\section{Translation duals}

If $O$ is a pseudo-ovoid consisting of $q^{2 n}+1(n-1)$-dimensional subspaces of PG $(4 n-1, q)$, then the tangent spaces of $O$ form a pseudo-ovoid $O^{*}$ in the dual space of $\operatorname{PG}(4 n-1, q)$; see Payne and Thas [9] (Theorem 8.7.2). The pseudo-ovoid $O^{*}$ is called the translation dual of $O$. If $q$ is even, then for every known pseudo-ovoid $O$ we have $O \cong O^{*}$; for $q$ odd, there are examples with $O \neq O^{*}$, see e.g. Payne [8]. Now we extend the notion of translation dual to semi-pseudo-ovoids.

Lemma 4.1 Let $O=\left\{\pi_{1}, \pi_{2}, \ldots, \pi_{\eta}\right\}$, with $\eta=1+q^{\frac{h+1}{2}}$, be a semi-pseudo-ovoid in $\mathrm{PG}(h, q)$ and let $\tau_{i}$ be the tangent space of $O$ at $\pi_{i}, i=1,2, \ldots, \eta$. If $\gamma$ is a hyperplane of $\tau_{i}$ not containing $\pi_{i}$, with $i \in\{1,2, \ldots, \eta\}$, then there is at least one $\tau_{j}$, with $j \neq i$, for which $\tau_{j} \cap \gamma$ is $(h-2 n)$-dimensional, that is, for which $\tau_{j} \cap \gamma=\tau_{i} \cap \tau_{j}$.

Proof: Assume, by way of contradiction, that for any $j \neq i$ we have that $\tau_{j} \cap \gamma$ is $(h-2 n-1)$-dimensional. Now we count in two ways the number of pairs $\left(z, \tau_{j}\right)$, with 
$z \in \gamma-\pi_{i}, z \in \tau_{j}$, and $j \neq i$. We obtain

$$
\frac{q^{h-n}-q^{n-1}}{q-1} \cdot q^{\frac{h-2 n+1}{2}}=q^{\frac{h+1}{2}} \cdot \frac{q^{h-2 n}-1}{q-1},
$$

clearly a contradiction.

Theorem 4.2 Let $O$ be a semi-pseudo-ovoid in $\operatorname{PG}(h, q)$, with $|O|=1+q^{\frac{h+1}{2}}$. Then the tangent spaces of $O$ form a semi-pseudo-ovoid $O^{*}$ in the dual space of $\mathrm{PG}(h, q)$.

Proof: Let $O=\left\{\pi_{1}, \pi_{2}, \ldots, \pi_{\eta}\right\}$, with $\eta=1+q^{\frac{h+1}{2}}$, and let $\tau_{i}$ be the tangent space of $O$ at $\pi_{i}, i=1,2, \ldots, \eta$. By Lemma 4.1 the space $\pi_{i}$ is the intersection of all hyperplanes $\gamma$ of $\tau_{i}$, for which the space $\left\langle\gamma, \tau_{j}\right\rangle$ generated by $\gamma$ and $\tau_{j}$ is $\operatorname{PG}(h, q)$, for all $j \neq i$. It follows that the tangent spaces of $O$ form a semi-pseudo-ovoid $O^{*}$ in the dual space of $\mathrm{PG}(h, q)$.

The semi-pseudo-ovoid $O^{*}$ will be called the translation dual of the semi-pseudoovoid $O$.

\section{Particular semi-pseudo-ovoids}

\section{1. $\alpha$-Regular semi-pseudo-ovoids}

A semi-pseudo-ovoid $O$ in $\operatorname{PG}(h, q)$ is called $\alpha$-regular if any $n$-dimensional subspace containing any element $\pi \in O$ but not contained in the tangent space of $O$ at $\pi$, has a point in common with exactly $\alpha$ elements of $O-\{\pi\}$. Any pseudo-oval and pseudo-ovoid is 1-regular. $\alpha$-Regular semi-ovals were studied in Blokhuis and Szönyi [1].

It is easily deduced, by considering all $n$-dimensional subspaces containing a given $\pi \in O$, that if the semi-pseudo-ovoid $O$ is $\alpha$-regular, then $|O|-1=\alpha q^{h-2 n+1}$.

Further Theorem 2.1 has an immediate corollary bounding $\alpha$.

Corollary 5.1 If $O$ is an $\alpha$-regular semi-pseudo-ovoid consisting of $\operatorname{PG}(n-1, q)$ in $\mathrm{PG}(h, q)$, then $\alpha \leq q^{2 n-\frac{h+1}{2}}$.

Proof: Since $|O|-1=\alpha q^{h-2 n+1}$, and $|O|-1 \leq q^{\frac{h+1}{2}}$ by Theorem 2.1, we obtain $\alpha \leq q^{2 n-\frac{h+1}{2}}$.

\subsection{SPG-reguli satisfying the polar property}

An SPG-regulus is a set $\mathcal{R}$ of $(n-1)$-dimensional subspaces $\pi_{1}, \ldots, \pi_{r}, r>1$, of $\mathrm{PG}(h, q)$, satisfying:

(a) $\pi_{i} \cap \pi_{j}=\emptyset$ for all $i \neq j$. 
(b) If $\operatorname{PG}(n, q)$ contains $\pi_{i}$, then it has a point in common with 0 or $\alpha(\alpha>0)$ spaces in $\mathcal{R} \backslash\left\{\pi_{i}\right\}$. If $\operatorname{PG}(n, q)$ contains $\pi_{i}$ and has no point in common with $\pi_{j}$ for all $j \neq i$, then it is called a tangent of $\mathcal{R}$ at $\pi_{i}$.

(c) If the point $x$ of $\mathrm{PG}(h, q)$ is not contained in an element of $\mathcal{R}$ it is contained in a constant number $\theta(\theta \geq 0)$ of tangents of $\mathcal{R}$.

SPG-reguli were introduced by Thas in [12] and give rise to semipartial geometries.

An SPG-regulus satisfies the polar property if $h>2 n-1$ and the union of tangents at each element $\pi_{i}$ of $\mathcal{R}$ is a $\mathrm{PG}^{(i)}(h-n, q)=: \tau_{i}(i \in\{1, \ldots, r\})$ which will be called the tangent space of $\mathcal{R}$ at $\pi_{i}$; see De Winter and Thas [4]. Clearly SPG-reguli satisfying the polar property are exactly $\alpha$-regular semi-pseudo-ovoids $O$, such that the number of tangent spaces on any point not in an element of $O$, is a constant.

Theorem 5.2 A semi-pseudo-ovoid $O$ is an SPG-regulus satisfying the polar property if and only if $|O|=1+q^{\frac{h+1}{2}}$.

Proof: First, suppose that $O$ is an SPG-regulus satisfying the polar property. Then $|O|=$ $1+q^{\frac{h+1}{2}}$ by Thas [12].

Next, suppose that $O$ is a semi-pseudo-ovoid satisfying $|O|=1+q^{\frac{h+1}{2}}$. Let $O=$ $\left\{\pi_{1}, \pi_{2}, \ldots, \pi_{\eta}\right\}$ and let $\tau_{i}$ be the tangent space of $O$ at $\pi_{i}, i=1,2, \ldots, \eta$. Further, let $\operatorname{PG}(n, q)$ contain $\pi_{i}$, with $\operatorname{PG}(n, q) \not \subset \tau_{i}$. Let $\alpha$ be the number of elements of $O-\left\{\pi_{i}\right\}$ intersecting $\mathrm{PG}(n, q)$. Now we count in two ways the number of pairs $\left(\pi_{j}, \phi\right)$, with $\pi_{j} \subset \phi$, $j \neq i, \phi$ a hyperplane containing $\operatorname{PG}(n, q)$. We obtain

$$
\alpha \frac{q^{h-2 n+1}-1}{q-1}+\left(q^{\frac{h+1}{2}}-\alpha\right) \frac{q^{h-2 n}-1}{q-1}=\frac{q^{h-n}-q^{n-1}}{q-1} \cdot q^{\frac{h-2 n+1}{2}} .
$$

Hence $\alpha=q^{2 n-\frac{h+1}{2}}$. As $\alpha$ is independent from $i$ and the choice of $\operatorname{PG}(n, q)$, it follows that $O$ is an SPG-regulus.

The problem on weak eggs in $\mathrm{PG}(4 n-1, q)$ mentioned in Section 2.2 now generalizes in a natural way to the following problem. Suppose $O=\left\{\pi_{1}, \pi_{2}, \ldots, \pi_{\eta}\right\}$ is a set of $\eta=1+q^{\frac{h+1}{2}}$ mutually disjoint $(n-1)$-dimensional spaces in $\operatorname{PG}(h, q)$. Further suppose that every $n$-dimensional space containing $\pi_{i}$, with $i=1,2, \ldots, \eta$, intersects either 0 or $\alpha=q^{2 n-\frac{h+1}{2}}$ elements of $O-\left\{\pi_{i}\right\}$. It is an open problem whether or not $O$ is a semi-pseudoovoid (and hence an SPG-regulus). Notice that for $h=4 n-1$ this problem is exactly the problem for weak eggs mentioned before.

Theorem 5.3 If $O$ is a semi-pseudo-ovoid with $|O|=1+q^{\frac{h+1}{2}}$, then the translation dual $O^{*}$ of $O$ is also an SPG-regulus satisfying the polar property.

Proof: Immediate from Theorems 4.2 and 5.2.

A generalized semi-pseudo-ovoid $O=\left\{\pi_{1}, \pi_{2}, \ldots, \pi_{\eta}\right\}$ in $\mathrm{PG}(h, q)$ is a set of $\eta$ mutually disjoint $(n-1)$-dimensional spaces in $\mathrm{PG}(h, q)$, with $h>2 n-1$, such that the union of the 
$n$-dimensional subspaces containing $\pi_{i}, i=1,2, \ldots, \eta$, and disjoint from all $\pi_{j}, j \neq i$, contains an $(h-n)$-dimensional space.

There is a variant of Theorem 5.2 for generalized semi-pseudo-ovoids that can be useful, as it is sometimes easy to check that on any $\pi_{i}$ there is an $(h-n)$-dimensional space $\tau_{i}$ disjoint from $\pi_{j}$, for every $j \neq i$, but difficult to check that every $n$-dimensional space containing $\pi_{i}$, but not contained in $\tau_{i}$, has non-empty intersection with $\tilde{O}-\pi_{i}$.

Theorem 5.4 Let O be a generalized semi-pseudo-ovoid in $\mathrm{PG}(h, q)$, with $|O|=1+q^{\frac{h+1}{2}}$. Then $O$ is an SPG-regulus satisfying the polar property.

Proof: Let $O=\left\{\pi_{1}, \pi_{2}, \ldots, \pi_{\eta}\right\}$ with $\eta=1+q^{\frac{h+1}{2}}$, and let $\tau_{i}$ be a fixed $(h-n)$ dimensional space containing $\pi_{i}$ and disjoint from $\pi_{j}, j \neq i$, for $i=1,2, \ldots, \eta$. Following the proof of Theorem 3.2 we see that every hyperplane containing no $\tau_{i}, i=1,2, \ldots, \eta$, contains exactly $1+q^{\frac{h-2 n+1}{2}}$ elements of $O$. Now let $\operatorname{PG}(n, q)$ be any $n$-dimensional space containing $\pi_{i}$ and having non-empty intersection with $\tilde{O}-\pi$. As in the proof of Theorem 5.2 we find that $\operatorname{PG}(n, q)$ intersects exactly $\alpha=q^{2 n-\frac{h+1}{2}}$ elements of $O-\left\{\pi_{i}\right\}$. We now easily obtain that there are exactly $\frac{q^{h-2 n+1}-1}{q-1} n$-dimensional spaces containing $\pi_{i}$ and having empty intersection with $\tilde{O}-\pi_{i}$. We conclude that $\tau_{i}$ is the union of all $n$-dimensional spaces containing $\pi_{i}$ and having empty intersection with $\tilde{O}-\pi_{i}, i=1,2, \ldots, \eta$, that is, $O$ is a semi-pseudo-ovoid. Applying Theorem 5.2 finishes the proof.

As an application we give a very short proof of a theorem of Luyckx [6] and provide a variant on Theorem 2.2, but first we give the definition of an $m$-system.

An $m$-system $\mathcal{M}$ of a finite (non-singular) classical polar space $P$ is a set, of maximal possible size, of mutually disjoint totally singular $m$-dimensional subspaces of $P$ with the property that no generator (that is, a maximal totally singular subspace) of $P$ that contains an element of $\mathcal{M}$ intersects any other element of $\mathcal{M}$. We have $|\mathcal{M}|=|P| / \mid$ generator $\mid$, as is shown in Shult and Thas [10] where $m$-systems were introduced.

Each $m$-system $\mathcal{M}$ of the polar space $P$, for which any $(m+1)$-dimensional subspace containing any $\pi \in \mathcal{M}$ and not contained in $\pi^{\perp}$ if $P$ is defined by a polarity, or not contained in the tangent space of $P$ at $\pi$ if $P$ is a quadric in even dimension over a field with characteristic two, has a point in common with at least one element of $\mathcal{M}-\{\pi\}$, provides an example of a semi-pseudo-ovoid.

Corollary 5.5 (Luyckx [6]) Let $O$ be an m-system of the polar space $P \in\left\{Q^{-}(2 n+\right.$ $\left.1, q), W_{2 n+1}(q), H(2 n, q)\right\}$, but not a spread of $W_{2 n+1}(q)$. Then $O$ is an $S P G$-regulus of the ambient space of $P$ satisfying the polar property.

Proof: If we denote the ambient space of $P$ as $\operatorname{PG}(h, q)$ then in each case there holds $|O|=1+q^{\frac{h+1}{2}}$. Furthermore, the definition of an $m$-system implies immediately that $O$ is a generalized semi-pseudo-ovoid. The result now follows from Theorem 5.4. 
The following variant of Theorem 2.2 shows that in the original definition of pseudoovoid $O(n, 2 n, q)$ the restriction that every three distinct elements of $O(n, 2 n, q)$ should generate a $\mathrm{PG}(3 n-1, q)$ is superfluous.

Corollary 5.6 Let $O$ be a generalized semi-pseudo-ovoid consisting of $1+q^{2 n}$ mutually disjoint $(n-1)$-dimensional spaces in $\mathrm{PG}(4 n-1, q)$. Then $O$ is a pseudo-ovoid.

Proof: Theorem 5.4 implies that $O$ is an SPG-regulus with $\alpha=1$. Hence every three distinct elements of $O$ generate a $(3 n-1)$-dimensional space, that is, $O$ is a pseudo-ovoid.

\section{Derivation of semi-pseudo-ovoids}

In this final section we show how new semi-pseudo-ovoids can be constructed from old ones without changing the size of the semi-pseudo-ovoid.

Theorem 6.1 Let $O=\left\{\pi_{1}, \pi_{2}, \ldots, \pi_{\eta}\right\}$ be a semi-pseudo-ovoid consisting of $(n-1)$ dimensional spaces in $\mathrm{PG}(h, q)$, with $\eta=1+q^{\frac{h+1}{2}}$. Let $\tau_{i}$ be the tangent space of $O$ at $\pi_{i}$. Suppose that the tangent spaces $\tau_{1}, \tau_{2}, \ldots, \tau_{s}$ have a $\mathrm{PG}(h-2 n, q)=: \Pi$ in common. If $\left\{\overline{\pi_{1}}, \overline{\pi_{2}}, \ldots, \overline{\pi_{s}}\right\}$ is a set of mutually disjoint $(n-1)$-dimensional spaces covering exactly the same point set as $\pi_{1} \cup \pi_{2} \cup \cdots \cup \pi_{s}$, then $\bar{O}=\left(O \cup\left\{\overline{\pi_{1}}, \overline{\pi_{2}}, \ldots, \overline{\pi_{s}}\right\}\right)-\left\{\pi_{1}, \pi_{2}, \ldots, \pi_{s}\right\}$ is also a semi-pseudo-ovoid and hence an SPG-regulus satisfying the polar property.

Proof: Clearly $\tau_{i}$ has empty intersection with the elements of $\bar{O}-\left\{\pi_{i}\right\}$, if $i \notin\{1,2, \ldots, s\}$. Furthermore it is obvious that the $(h-n)$-dimensional space $\left\langle\bar{\pi}_{j}, \Pi\right\rangle$ has empty intersection with the elements of $\bar{O}-\left\{\bar{\pi}_{j}\right\}, j=1,2, \ldots, s$. We conclude that $\bar{O}$ is a generalized semi-pseudo-ovoid with $|\bar{O}|=1+q^{\frac{h+1}{2}}$. Theorem 5.4 finishes the proof.

This theorem generalizes a result from De Winter and Thas [4], where this is shown to be true if $O$ is a set of $1+q^{3}$ lines in $\operatorname{PG}(5, q)$ arising from a Buekenhout-Metz unital in $\mathrm{PG}\left(2, q^{2}\right)$. It is also not so difficult to see that it is a generalization of a result of Luyckx and Thas [7] on derivation of $m$-systems as well.

\section{References}

1. A. Blokhuis and T. Szönyi, "Note on the structure of semiovals in finite projective planes, A collection of contributions in honour of Jack van Lint," Discrete Math. 106/107 (1992), 61-65.

2. F. Buekenhout, "Characterizations of semi quadrics. A survey," Colloquio Internazionale sulle Teorie Combinatorie (Roma, 1973), Tomo I, pp. 393-421. Atti dei Convegni Lincei, No. 17, Accad. Naz. Lincei, Rome, 1976.

3. R. Calderbank and W.K. Kantor, "The geometry of two-weight codes," Bull. London Math. Soc. 18(2) (1986), 97-122.

4. S. De Winter and J.A. Thas, "SPG-reguli satisfying the polar property and a new semipartial geometry," Des. Codes Cryptogr. 32 (2004), 153-166. 
5. M. Lavrauw, "Scattered spaces with respect to spreads, and eggs in finite projective spaces," Dissertation, Eindhoven University of Technology, Eindhoven, 2001, viii+115 pp.

6. D. Luyckx, "m-systems of polar spaces and SPG reguli," Bull. Belg. Math. Soc. Simon Stevin 9(2) (2002), 177-183.

7. D. Luyckx and J.A. Thas, "Derivation of $m$-systems," European J. Combin. 24(2) (2003), 137-147.

8. S.E. Payne, "An essay on skew translation generalized quadrangles," Geom. Dedicata 32(1) (1989), 93-118.

9. S.E. Payne and J.A. Thas, Finite Generalized Quadrangles, Research Notes in Mathematics, vol. 110, Pitman Advanced Publishing Program, Boston, MA, 1984, vi+312 pp.

10. E.E. Shult and J.A. Thas, "m-systems of polar spaces," J. Combin. Theory Ser. A 68(1) (1994), 184-204.

11. J.A. Thas, "On semi-ovals and semi-ovoids," Geometriae Dedicata 3 (1974), 229-231.

12. J.A. Thas, "Semipartial geometries and spreads of classical polar spaces," J. Combin. Theory Ser. A 35 (1983), 58-66. 